Mots. Les langages du politique

\title{
L'émotion dans les médias : dispositifs, formes et
} figures

Emotion in mass media: devices, forms and figures

La emoción en los mass media : dispositivos, formas, figuras

Jean-François Tétu

\section{OpenEdition}

Journals

Édition électronique

URL : https://journals.openedition.org/mots/2843

DOI : $10.4000 /$ mots. 2843

ISSN : 1960-6001

Éditeur

ENS Éditions

Édition imprimée

Date de publication : 1 juillet 2004

Pagination : 9-20

ISBN : 2-84788-057-7

ISSN : 0243-6450

\section{Référence électronique}

Jean-François Tétu, «L'émotion dans les médias : dispositifs, formes et figures », Mots. Les langages

du politique [En ligne], 75 | 2004, mis en ligne le 22 avril 2008, consulté le 22 avril 2022. URL : http:// journals.openedition.org/mots/2843; DOl : https://doi.org/10.4000/mots.2843

\section{(C) ENS Éditions}


Jean-François TÉTU ${ }^{1}$

\section{L'émotion dans les médias: dispositifs, formes et figures}

L'émotion, omniprésente aujourd'hui dans les médias, notamment à la télévision qui fait se succéder devant nos yeux les conflits, les catastrophes et les agressions, pose des questions spécifiques. Et d'abord parce que l'émotion suspend d'emblée le raisonnement («je le vis, je rougis, je pâlis à sa vue»). En cela, et parce qu'elle repose sur une perception (voir infra la place spécifique du regard), elle semble se détourner de la rationalité constitutive de l'espace public comme espace de débat. Sauf que nous savons très bien, depuis Voltaire et l'affaire du chevalier de La Barre, que l'opinion peut se mobiliser pour défendre des causes qui lui sont rendues sensibles par le spectacle d'une souffrance indue, prendre le parti des victimes supposées injustement maltraitées (Sacco et Vanzetti), ou dénoncer des «bourreaux», responsables directs ou lointains de la souffrance d'autrui (affaire Dreyfus, etc.).

Comment peut-on rendre compte d'un processus médiatique qui repose sur la perception d'une situation extérieure aux récepteurs de l'information et conduit, selon les cas, à un attendrissement partagé (couronnement d'un souverain), à un déferlement de liesse populaire (la France victorieuse au Mondial de football en 1998), à une mobilisation charitable (l'abbé Pierre en 1954) ou à une mobilisation politique (expulsion des sans-papiers de l'église Saint-Bernard)? À quoi il faut ajouter que, parfois, l'opinion reste inerte (bombardement d'Hiroshima).

Il est possible d'esquisser une réponse en examinant cette question sous trois angles: celui du dispositif même de l'information; celui de sa thématique; celui de sa rhétorique ou de sa mise en scène discursive.

On voit bien qu'il est impossible d'examiner ce problème sans prendre en compte la redoutable question des effets. Nous n'évoquerons pas ici la réception de l'information, processus qui est pourtant indispensable à leur compréhension. Nous partons du seul principe que le langage des médias (tout à la fois dispositif de mise en page ou en scène, image et texte) fait sens dans

1. Institut d'études politiques - Université Lumière - Lyon 2 - jean-francois.tetu@univ-lyon2.fr 
une relation de communication aux trois niveaux précités. Cet article a donc trois limites fortes: il n'envisage l'émotion que comme révélateur d'un certain nombre de dispositifs et de procédés dont il esquisse l'analyse; il ne traite pas de l'intégralité des médias mais de la seule information, elle-même limitée à la télévision au départ, puis à l'écrit; enfin, écartant la question des effets, il laisse de côté le sujet de l'émotion: il est, en effet, indiscutable que l'on n'est ému qu'en fonction de ce qu'on est, si bien que chaque média organise sa propre rhétorique en fonction de l'image qu'il se forme de son public (comme en témoigne par exemple l'inflation du discours direct dans la presse populaire).

\section{Le dispositif}

Nous examinerons ici le seul cas de la télévision, parce que la télévision donne d'abord à voir (le son est monté après l'image). Et il semble bien qu'il y ait un lien fondamental entre l'émotion et la vue. Certes, si l'émotion, issue d'une perception, peut a priori surgir de chacun de nos sens (un bruit inattendu fait sursauter), la vue semble bénéficier à cet égard d'une place originale: témoin, de façon archétypale, la cécité de Tirésias et d'CEdipe à Colone, qui leur permet d'échapper aux apparences pour exercer leur raison; ou le mythe de la caverne; ou encore le Bon Samaritain - qui peut servir de modèle à toute posture humanitaire - se détournant lorsqu'il voit un blessé. L'image, pendant des siècles, a bénéficié d'une fonction édifiante dans l'iconographie profane ou religieuse.

L'information télévisée propose chaque jour au téléspectateur une situation reconnaissable. Celle-ci met en jeu une norme sociale dans laquelle un actant est montré comme victime ou bénéficiaire. Le cas le plus fréquent est celui de la description d'une scène dramatique : à la suite de l'éruption du volcan Nevado del Ruiz, un reportage montre l'agonie de la petite Omeyra, prise dans une gangue de boue sans que le photographe puisse lui porter secours. Aucun commentaire de la part du présentateur (Noël Mamère, qui présentait le JT à 13 heures ce jour-là, nous a dit avoir pris le parti de se taire parce qu'il découvrait la scène en même temps que le téléspectateur). L'absence de commentaire renforce ici l'effet supposé sur le spectateur. Le second cas est celui où le présentateur explique la situation en indiquant l'état dans lequel est supposé se trouver le locuteur (par exemple, la victime d'un attentat ou d'un accident) ou le téléspectateur lui-même, sur le mode: «ayez peur» (de ce nuage toxique), «ayez pitié» (de ces malheureux). Les deux actants peuvent être réunis, comme dans le fameux «la France a peur» de Roger Gicquel, lors de l'enlèvement du petit Philippe Bertrand. 
Le cas le plus spécifique à la télévision est celui du direct. On en a beaucoup dénoncé les effets désastreux sur l'information, et plus encore sur l'information en continu, qui n'offre aucun recul par rapport à l'évènement. C'est bien pour cela que le direct est propice au surgissement de l'émotion. Il vise un effet de présence. Mais, alors qu'il peut être fortement narrativisé dans le faux direct ou d'usage systématique dans les informations radiophoniques, le reportage en direct, même très narrativisé comme peut l'être la retransmission d'une compétition sportive, laisse une place capitale à l'imprévu: accident dans les stades du Heysel ou de Furiani, chute du peloton dans un sprint final, sortie de piste d'une automobile en compétition. La question ici est de savoir si la fascination du direct, que nous tenons pour acquise tant elle est promue et revendiquée, a un lien particulier avec la production des émotions.

Dans Media Events, Elihu Katz et Daniel Dayan remarquent que le spectateur, notamment dans les cérémonies programmées, est appelé à se transformer en témoin. Quel genre de témoin? Non pas le

témoin objectif de la science empirique, le vérificateur dont le regard silencieux et froid se confondait avec l'exercice de la raison, [mais] un témoin au sens que prenait ce terme dans le christianisme antique, celui à qui on demande de se convertir à une nouvelle définition de la réalité, puis de lui servir de média, de devenir l'instrument de sa propagation ${ }^{2}$.

Cette analyse constitue une première réponse: l'information ne cherche pas ici un savoir ni même un voir, mais un faire-voir susceptible de produire directement un croire, indispensable à l'émotion: «j'y étais [devant la TV], je l'ai vu». Le direct produit l'expérience d'un présent insaisissable, irreprésentable comme présent parce que, à peine perçu, il est déjà passé; mais pour cette raison même le direct est propice à la propagation des émotions, car il reste à raconter et à reraconter. Il permet de passer d'une émotion comme suspens du sens à une parole qui lui re-donne sens. Mais cette parole-là, secondaire, appartient au spectateur qui trouve dans le direct un rôle, un programme d'action (l'action ici est d'en parler). Il projète donc chez le spectateur un programme virtuel de prise de parole; ce n'est pas l'émetteur mais le récepteur à qui est déléguée la fonction, virtuelle, du commentaire. L'interprétation de l'action est transférée au spectateur qui peut la reconstruire. Cela nous semble une explication plausible de l'attrait du direct, qui laisse le champ libre au récepteur pour sa propre refiguration.

2. E. Katz et D. Dayan, 1996, La télévision cérémonielle, Paris, PUF, p. 132 (traduit de: 1992, Media Events, Cambridge, Harvard University Press).

3. J.-F. Tétu, 1994, «La radio, ou la maitrise du temps», Bulletin du Certeic, $\mathrm{n}^{\circ} 15$, Études de communication, p. 86. 
Une autre explication de l'effet du direct est possible : elle recourt à l'usage que faisait Jacques Lacan du concept de réel ${ }^{3}$. Le réel, pour Lacan, c'est ce qui est dans l'ordre de la cause. Celle-ci tiendrait à l'ordre des choses: ainsi la chute d'un corps, du fait de la gravitation universelle. La chute de ce corps, serait-ce une tribune dans un stade, n'a en soi aucun sens; mais le discours que l'on peut tenir à son propos permet de lui en trouver un. Bref, - et on nous pardonnera ce raccourci de la pensée de Lacan - le réel est proprement irreprésentable, parce que, régi par la cause, il est insensé, alors que la construction de nos représentations n'a qu'un but, l'organisation des significations.

Je ne peux pas regarder, à la télévision, un match de tennis ou de football sans éprouver quelque chose comme une passion du coup qui viendra: $\mathrm{j}$ 'attends, passivement, de subir l'émotion du coup attendu ou inattendu. Si le coup est attendu (c'est-à-dire s'il correspond au schéma narratif prévu), je puis ressentir la joie du supporter, mais aucun trouble ne me guette. Je n'éprouve que le plaisir d'une répétition: le spectacle vient me fournir le double de ce que j'avais préconçu. Ce qui me passionne, en revanche, c'est l'attente de ce qui, n'étant pas préconçu, va bientôt faire parler (et que, en tant que supporter, je puis aussi bien espérer que redouter); bref, ce qui, dans le programme, peut échapper au programme. Donc, j'attends. J'anticipe, je scénarise, je pré-récite autant que la régie, sur fond d'images produites par la régie, mais j'attends confusément quelque chose.

Quand l'inattendu survient (le tennisman glisse, et perd; Challenger explose en vol), je me trouve devant une image, une représentation, qui me renvoie à ce qui demeure pour moi proprement insensé et irreprésentable, alors que dans un film-catastrophe, la catastrophe est tellement pré-construite qu'elle ne fait surgir aucun insensé, mais de l'héroïsme ou de la turpitude à foison. En somme, la force du direct, c'est qu'il permettrait l'attente de l'irreprésentable, quitte à construire, ensuite, de grands édifices représentatifs à son sujet. Attente de l'irreprésentable, du moment où le réel lacanien surgit dans le parcours pré-raconté. Dans le direct, le téléspectateur fait une expérience originale de suspens du sens, parce que tant de significations se révèlent possibles qu'il ne sait pas a priori laquelle est la bonne, même si l'angle de prise de vue, par exemple, constitue le point d'où il voit les choses. Bref, parce que c'est au présent et que le présent échappe, il perçoit que la cause peut prendre la place du sens, ce qui est une source majeure d'émotion.

La fonction de l'image d'information a connu depuis quelques décennies une forte modification, due à la multiplication des échanges internationaux de documents filmés. Cela aboutit à une standardisation des informations télévisées, comme il apparait lorsque la source des images est raréfiée (guerre du Golfe), mais aussi à une décontextualisation qui nous semble à l'origine d'une forme spécifique d'émotion. On le sait bien depuis Roland Barthes : le mode original 
de signification de l'image photographique est de combiner l'«ici-maintenant» de la lecture et l' "'avant-ailleurs» de la prise. L'image d'actualité, même lorsqu'elle n'est pas en direct, produit une concordance des temps (le présent de l'actualité) en même temps qu'une discordance radicale entre le contexte d'origine et le contexte de réception. Cela aboutit à une rupture entre l'information et l'action, entre l'ici du récepteur et le là-bas du conflit ou de la catastrophe. Si l'émotion est bien «ce qui déplace», nous avons affaire à une émotion particulière, celle qui appelle une réaction impossible du fait de la discordance des lieux. Elle conduit soit à une action décalée, par exemple l'expédition de sacs de riz en Somalie, soit à une impossibilité d'action, qui fait de l'émotion le cœur même de la consommation médiatique.

Précisons. Les images des situations catastrophiques sont légion. Elles sont comme les matériaux bruts d'une situation insupportable et presque indicible (le commentaire est d'autant plus sobre que la mort est plus visible et, en fait, irreprésentable) : la mort est évidemment là. D'où le quasi-silence du commentaire. Cela distingue radicalement le direct télévisé du direct radiophonique où les voix sont surabondantes (commentateur sportif haletant ou paroles des rescapés d'une catastrophe), et le silence très perturbant. À la radio, l'évènement n'est que voix ou son, et cette condensation synecdochique produit nécessairement chez l'auditeur un appel à l'expansion symétrique des représentations. Pourquoi ne rien dire alors aux moments les plus intenses de l'image télévisée? Parce qu'il existe une discordance insurmontable entre l'évidence du constat et l'impossibilité de changer le cours de l'action insupportable: les images que reçoit le téléspectateur sont, absolument, différentes ou séparées de la vision in situ des acteurs de l'évènement.

Mais autre chose mérite d'être examiné. Devant chaque catastrophe, les médias soulignent la solidarité de la population. Or, cette solidarité sur le terrain est interdite au téléspectateur. L'action solidaire immédiate peut être impossible à quiconque (explosion de la navette Challenger) ou inutile (mort de Lady Di). Elle peut aussi n'être que déléguée (catastrophe lointaine). Il reste alors un écart insurmontable entre les acteurs (sur place) et les téléspectateurs (au loin). D'où la prolifération des dispositifs d'aide d'urgence : leur montée coïncide avec la visibilité des catastrophes d'actualité. L'image court-circuite les dispositifs institutionnels du contexte, elle vise une urgence qui rend le discours inutile et pousse à agir tout de suite avec des médiateurs eux aussi urgents («médecins d'urgence»). Tant qu'il y a des vies à sauver, l'humanité peut trouver dans l'horreur un aliment et une justification. En d'autres termes, la solidarité des gestes humanitaires est une réponse à une émotion inassimilable, celle qui nous ferait spectateurs tranquilles de la mort ou de la douleur d'autrui. 
La mondialisation de l'information a stimulé l'action humanitaire, non comme une réponse politique à des actions injustifiables (Rwanda, Kosovo ou Tchétchénie), mais comme seule réponse du téléspectateur à ce qu'il reçoit de loin. L'urgence humanitaire, de date récente, doit beaucoup aux médias parce que la vision de la catastrophe appelle une réaction immédiate, en réponse à la médiatisation de l'émotion qu'elle provoque.

\section{Les thématiques}

Depuis plus d'un siècle, la psychologie a défini l'émotion comme un trouble de l'adaptation ${ }^{4}$. Le fait que le sujet ne dispose pas d'une réponse toute prête perturbe son activité et ses représentations. Le lien a souvent été fait entre l'émotion et la motivation (même origine langagière: le latin movere). Mais là où le bon samaritain, ému par la détresse du blessé, se détourne pour lui porter secours, l'usager des médias, lui, n'a aucune possibilité d'action immédiate, parce qu'il n'est pas dans la situation dont il n'est qu'un lointain témoin: il ne peut pas fuir Manhattan. Puisqu'il est dans l'ordre des représentations, la seule émotion possible devrait être de nature esthétique; or nous savons que ce n'est pas le cas. L'information, pour un temps au moins, doit faire croire que l'usager des médias se trouve confronté à une situation émouvante.

Une situation émouvante, dans la réalité, tient à la nature de la relation entretenue avec le sujet et provoquant peur ou colère, joie ou honte, etc. Il faut au support d'information quelque chose qui soit commun au groupe social: il doit représenter une situation à propos de laquelle une norme sociale (un jugement de valeur socialement partagé) met en cause dans un récit un actant qui est, en général soudainement, victime ou bénéficiaire. Cette situation doit en outre reposer sur une motivation forte. D'où le succès de représentations qui renvoient à des expériences communes, comme le trac avant un examen ou le saisissement devant une épreuve. Le sport déploie ainsi les réactions affectives communément possibles, comme l'angoisse du gardien de but avant le pénalty ou l'explosion de liesse après un essai. Comme l'émotion ne va pas sans les sentiments qui l'accompagnent ou la suivent, c'est par le brandissement des signes les exprimant que la situation est rendue émouvante: «Le monde a peur», titre Le Parisien du 12 septembre 2001. Nous l'avons appris depuis l'enfance: les sentiments peuvent se communiquer plus facilement lorsque l'on en amplifie

4. John Dewey: «Je vois un ours, je tremble, j'ai peur» (au lieu de la proposition classique: «Je vois un ours, j'ai peur, je tremble»). Voir «The Theory of Emotion», 2, «The Significance of Emotions », Psychological Review, 1895 (l'Encyclopaedia Universalis attribue cette phrase à William James, 1884). 
les signes. La dramatisation est une constante de la médiatisation de l'émotion, sous des formes propres à chaque support.

La dramatisation des sentiments est donc le premier ressort thématique de la représentation des émotions. Le deuxième, qui va de pair avec lui au point d'en être quasiment indistinct, est l'évènementialisation, c'est-à-dire l'exhibition d'une fracture dans l'état du monde. C'est bien ce qu'exprime le recours, au soir du 11 septembre, à l'expression la guerre (Paris Match, Marianne, L'Express, Le Figaro), ou aux vocables qui indiquent un choc: l'épouvante (La Croix), l'Amérique foudroyée (Les Échos). Cela montre la volonté des informateurs de mettre le public dans une sorte de prise directe sur l'évènement, qui conduit à exhiber en titre tantôt une posture compassionnelle (l'épouvante), tantôt la dénonciation des bourreaux (les nouveaux barbares, dans Marianne). Ce sont là les principaux régimes de discours, qu'on retrouve lorsqu'une catastrophe alimente l'actualité: naufrage du Prestige et marée noire inéluctable, milliers d'automobilistes bloqués sur une autoroute par le verglas, etc. On distingue chaque fois trois composantes majeures:

- la norme sociale sous la forme de la morale à maintenir (solidarité à l'égard des victimes $v s$ dénonciation de profiteurs en tous genres, pillards ou voyeurs; respect des personnes et de la propriété);

- la norme sociale sous la forme de l'opinion, prompte à dénoncer l'inaction, le retard ou l'inefficacité des pouvoirs publics ou des autorités. On constate en particulier une tendance forte des médias à prendre parti pour la victime, afin de susciter l'identification du lecteur (l'opinion se fonde sur la morale précédente);

- l'esthétisation du spectacle de la désolation: pietà algérienne à la suite d'un massacre. Cette esthétisation participe à la fois de la thématique et de la figuration.

La compassion et la dénonciation ${ }^{5}$ peuvent prendre d'autres formes: celle de l'attendrissement devant l'action bienfaisante et, à l'opposé, celle de l'indignation qui présuppose l'existence d'un monde juste (Luc Boltanski). L'appel à la justice permet d'articuler la dénonciation du malheur et l'accusation des auteurs présumés du même malheur: la folie des hommes ou leur inhumanité (Dutroux, Ben Laden, les acteurs des guerres civiles). Ce qui explique les deux figures majeures du thème de la pitié: d'une part, le malheur, lorsqu'il est renvoyé au coupable/responsable, conduit à la mise en scène du ressentiment de la victime

5. L. Boltanski, 1993, La souffrance à distance, morale humanitaire, médias et politique, Paris, Métaillé, p. 15-37 et p. 91-116. Voir également P. Charaudeau, 2000, «Une problématisation discursive de l'émotion. À propos des effets de pathémisation à la télévision», C. Plantin (dir.), Les émotions dans les interactions, Lyon, PUL, p. 125-156. 
(«je n'ai plus rien», dit la victime) et, de ce fait, à l'indignation du (télé-)spectateur; d'autre part, le malheur, lorsqu'il est combattu par le bienfaiteur, conduit à la gratitude de la victime et à l'attendrissement du spectateur. Ce cas, plus rare, est surtout le fait des catastrophes naturelles où la culture (solidarité des hommes) s'oppose à la violence aveugle de la nature. Une figure de cet ordre, assez peu fréquente dans l'information, appartient à un autre registre de la télévision, celui des reality-shows initiaux ou des magazines (tel Perdu de vue).

Entre la victime du monde «réel» et l'usager des médias, un tiers est nécessaire. Roland Barthes en a fait une analyse magistrale, dans Mythologies, avec la figure de l'abbé Pierre ${ }^{6}$. Ce qui montre que le réalisme a un effet mythique immédiat: soustraire la souffrance à une victime pour l'exhiber devant les usagers des médias qui, eux, ne souffrent pas.

La troisième thématique majeure, issue de la précédente, est la menace sur la sociabilité. D'où l'insistance sur l'opposition guerre/paix comme figure archétypale du bien ou du mal-être social. Il s'agit ici, surtout, de guerre interne au corps social: le chauffard meurtrier qui est un élu local, le voisin qui peut être un terroriste, etc. C'est ainsi que le conflit de Bosnie fut d'abord montré: on se rappelle par exemple le jeune couple dont l'un des membres était bosniaque et l'autre serbe et qui fut assassiné sur un pont de Sarajevo, sans que personne n'ose recueillir les cadavres. Tout ce qui bloque le social normal mobilise fortement les affects : autoroute coupée, aéroport fermé, grande panne de téléphone ou d'électricité.

La dernière thématique est celle de la menace sur l'humanité, d'où le recours à la figure majeure du «monstre», source d'une menace latente pour chacun, afin d'expliquer l'inexplicable. C'est une des figures les plus anciennes des médias, dont les faits divers sont remplis (passage miraculeux d'une armée d'anges dans le ciel $v s$ crimes abominables).

Ces grands axes thématiques: la fracture et l'excès, l'asocial et l'inhumain fournissent ainsi des figures renouvelées sans cesse et permettent la mise en œuvre de procédés rhétoriques récurrents.

\section{La mise en scène discursive}

La rhétorique des émotions n'est pas, pour une large part, spécifique: elle ne fait qu'amplifier les traits habituels de toute information et utilise toutes les figures possibles de la rhétorique traditionnelle. On peut cependant en repérer les tendances majeures, que nous relèverons dans la seule information écrite.

6. R. Barthes, 1957, Mythologies, Paris, Seuil, rééd. 1970, p. 54. 
La première est le minimalisme ou la minimalité du discours, que l'on pourrait aussi définir comme une raréfaction des mots. Ainsi, en 1914 ou 1939 : «Mobilisation générale» ou «La guerre». Et tout est dit. A contrario, après les accords de Munich, Paris-Soir Dimanche (dernière édition, le $1^{\text {er }}$ octobre 1938) ne comporte qu'un titre, «La Paix», quand Le Figaro, la veille, était plus bavard: «La paix est sauvée». Plus près de nous, l'élection présidentielle de 2002 est scandée dans Libération par trois interjections: «NON», «OUI», «OUF». C'est la forme rhétorique la plus proche de la stupeur suscitée par l'évènement, qui laisse sans voix, bouche bée. Il faut en dire le moins possible (litote) mais avec une recherche de la désignation la plus appropriée, qui, dans les deux cas précédents, renvoie, soit à l'expression de l'informateur comme archi-lecteur (Libération), soit à la référence (Paris-Soir), sous la forme la plus condensée.

Le choix des mots, quant à lui, semble reposer sur quatre stratégies ${ }^{7}$ : $1^{1}$ hyperbole (d'où l'inflation fréquente des chiffres); le choix de mots qui décrivent l'émotion de manière transparente (joie, peur, colère); le choix de mots supposés déclencher l'émotion (terroriste, tueur, violeur, etc.); le choix, enfin, d'énoncés qui produisent de l'émotion du fait de la situation. L'opérateur d'une maison de courtage raconte:

J'étais au téléphone avec une fille à New York et elle m'a dit: «L'immeuble est en train d'exploser». Depuis, je n'ai plus de nouvelles. [...] «Putain, nous sommes en train de mourir», hurle un inconnu avant de raccrocher... au moment où s'effondre sa tour. (Paris Match, après le 11 septembre)

Le «poids des mots» n'irait pas ici sans le «choc des photos» qui appartient le plus souvent aux registres suivants:

- celui d'une culture commune, largement empruntée à l'univers pictural: la femme couverte de cendres le 11 septembre, yeux fixes et bouche ouverte, semble sortie tout droit de Pompéi. Ici, la référence culturelle donne une sorte d'éternité instantanée à la victime, ou à l'évènement;

- celui, à l'opposé, du «jamais-vu» (l'explosion de Challenger), compréhensible seulement par l'usage de signes visuels (la fumée, le feu) qui renvoient à la catastrophe. La vague déferlante appartient au même registre qui signifie, par l'emprunt de signes naturels (l'eau ou le feu), que la nature submerge la culture;

- un autre registre est celui du geste tout à fait inattendu, qui renvoie à l'émotion de l'acteur photographié: le pape embrassant le sol, la première fois (ensuite, cela devient un geste rituel); Mitterrand et Kohl se tenant par la main, etc. Le caractère incongru ou inédit constitue une figure de la rupture évènementielle;

7. P. Charaudeau, art. cité, p. 139. 
- le dernier est celui de la forme hyperbolique d'un geste usuel (saut inimaginable de Noureev, embrassades spectaculaires de sportifs après un but), qui correspondant à la dramatisation que nous avons signalée.

La tentative de faire une sorte d'inventaire des figures les plus fréquentes dans la représentation des situations émouvantes est un peu vaine. Il en existe certes de privilégiées, de la litote à l'hyperbole, mais ce sont toutes les figures rhétoriques qui sont plus ou moins convoquées, de la métonymie banale (les cendres sur le visage d'un pompier) à la synecdoque (monceaux de ferrailles tordues en place du World Trade Center). À une réserve près: certaines figures (chiasme ou oxymore, par exemple) imposent une distance, une intellectualisation contradictoire avec le choc émotionnel, qui préfère l'anaphore, l'allitération, l'antithèse, l'ellipse, l'hyperbole, la répétition, la synecdoque, etc.

Une tendance privilégiée est la présentification, qui réduit l'écart temporel et spatial sur le modèle de «Madame se meurt, Madame est morte»: tous les titres et toutes les légendes du numéro spécial de Paris Match sur le 11 septembre sont soit des phrases nominales, soit des présents narratifs. Un moyen souvent mis en œuvre est le réalisme de la description: incipit in medias res, retard du sens, etc. ${ }^{8}$, avec l'omniprésence de la métonymie. Enfin, une figure clé de l'émotion est la référence à l' «onde de choc» de l'évènement, image privilégiée des médias qui se refusent généralement au sensationnalisme. On lit par exemple dans Les Échos du 12 septembre: «Le monde s'est figé d'effroi, après les attentats. [...] Et les messages ont afflué de la planète». Même chose à peu près dans La Croix: «inspirer la terreur au monde entier, et faire horreur à tous les habitants de la planète».

Une piste utile pour comprendre l'émotion médiatique a été proposée par Patrick Charaudeau, qui oppose le savoir de connaissance au savoir de croyance à l'œuvre dans l'émotion. Pour cela il faut, selon lui, à la fois une information (un savoir), une évaluation par rapport à ce savoir, et enfin une position, ce qui nous renvoie à la norme sociale ou au jugement de valeur que nous avons évoqués plus haut ${ }^{9}$.

La particularité de la situation médiatique est que là où, dans la réalité, il y aurait une obligation d'assistance, il ne peut exister pour l'usager des médias qu'une saturation de l'imagination. D'où l'intérêt de la pensée lacanienne pour cet objet: d'abord le surgissement du réel (l'avion explose du fait de l'impact; les tours s'effondrent du fait de la chaleur). Cela est proprement irreprésentable: aucune loi n'autorise l'assimilation de cet attentat, car nous sommes en période

8. M. Mouillaud et J.-F. Tétu, 1989, Le journal quotidien, Lyon, PUL, p. 157-158.

9. P. Charaudeau, art. cité, p. 130-131.

10. J.-F. Tétu, 1982, Le discours du journal, thèse, Université Lyon 2, tome 2. 
de paix, alors que les comptes rendus du bombardement d'Hiroshima n'ont suscité en leur temps aucune émotion particulière, sinon l'émoi du merveilleux ( «À quand le premier voyage sur la lune? ${ }^{10}$ ). Il ne reste à l'usager des médias que l'image de lui-même, confrontée à ce qu'il ne peut assimiler que comme fantasme.

Soit, pour conclure, la situation suivante: des pompiers volontaires, en train de porter assistance à un automobiliste accidenté, sont fauchés par une voiture qui roule à une vitesse folle. L'attention, très vite, se détourne des victimes pour se concentrer sur le «bourreau». C'est un cas, exemplaire, où l'on voit s'inverser l'attention accordée à la victime et à son persécuteur (Patrick Henry, Patrice Alègre, etc.). Il y aurait donc, en fait, une théorie du pouvoir sous-jacente qui fait juger de façon différente les victimes du verglas sur la route en hiver et les abandonnés de Cofiroute en janvier 2003, parce que, dans le second cas, on peut penser qu'une instance quelconque du pouvoir aurait pu éviter ce dérèglement.

Cette théorie sous-jacente peut seule expliquer la différence de traitement, selon les médias, de l'indignation et de l'apitoiement. Après le coup de grisou de Liévin, en 1971, où 40 mineurs trouvent la mort à 700 mètres sous terre, on peut trouver une pitié sans colère ("Cette faille était maudite», lit-on dans France Soir) ou une colère sans pitié ( $« 40$, ils en ont tué $40 »$, s'indigne Libération). Il y aurait ainsi une représentation critique implicite d'un pouvoir qui constituerait le véritable destinateur de l'action représentée: derrière le brandissement de l'émotion comme dénominateur commun de l'humanité, se trouverait un présupposé de nature politique, qui trouve dans une situation émouvante, euphorique ou dysphorique, une façon de dire l'ordre ou le désordre du monde. 